\section{Tampering with transcription}

\author{
Gary J. Nabel
}

Concealed within a cell, the human immunodeficiency virus (HIV) quietly subverts a host that would otherwise seek to destroy it. Productive HIV infection reflects a delicate balance between the needs of the host cell and the demands of the virus. It is now clear that this balance has not been left simply to chance - two studies, one that appeared in last week's Nature ${ }^{1}$, the other reported on page 709 of this issue ${ }^{2}$, show that sophisticated strategies have evolved to alter cellular transcription and facilitate viral replication. Many cellular proteins contribute to the replication of $\mathrm{HIV}$, including $\mathrm{NF}-\kappa \mathrm{B}$, an inducible transcription factor that binds to regulatory sites in the HIV enhancer sequence and increases viral transcription after $\mathrm{T}$-cell activation ${ }^{3}$. It now seems that NF- $K \mathrm{~B}$ is also specifically induced during the surse of infection ${ }^{1}$, and that it may be regulated by a viral gene product, the HIV-1 protease ${ }^{2}$.

Like all viruses, HIV is an intracellular parasite. Beginning with its entry into its host cell by way of the CD4 glycoprotein, the virus interacts with many cellular proteins to complete its life cycle. The importance of these host-cell factors became apparent during early attempts to isolate the virus. Propagation was much more successful when the host $\mathrm{T}$ cell was activated by mitogens $\mathrm{s}^{4,5}$, which causes an increase in NF- $K \mathrm{~B}$ binding to DNA; and the two $\kappa \mathrm{B}$ elements in the HIV-1 regulatory region, although not indispensable for replication ${ }^{6}$, are responsible for the increase in stimulation through the enhancer sequence seen in activated $T$ cells.

In cells infected by HIV, including T cells and some monocytes, NF- $K \mathrm{~B}$ resides within the cytoplasm in an inactive form, complexed to an inhibitor, $\mathrm{I}_{\kappa} \mathrm{B}$. Cellular activation by mitogens or cytokines releases the active DNA-binding complex, which is composed of distinct DNA-binding (p50) and transactivation (p65) subunits (see figure). This process is regulated at several levels phosphorylation by protein kinases, for example, causes $\mathrm{I} K \mathrm{~B}$ to disengage from the $\mathrm{p} 50 / \mathrm{p} 65$ complex $^{7}$. Another regulatory step probably involves the DNA-binding subunit of $\mathrm{NF}-\kappa \mathrm{B}$. This protein is synthesized as a precursor of relative molecular mass $105,000\left(M_{\mathrm{r}} 105 \mathrm{~K}\right)$ and requires processing to generate the active binding protein of $M_{\mathrm{r}} 50 \mathrm{~K}$.

Bachelerie and co-workers have now shown that infection by HIV stimulates binding of $N F-\kappa$ to $D^{-} A^{2}$. In a promonocytic line, infection leads to a marked increase in $\mathrm{NF}-\kappa \mathrm{B}$ binding activity and function, raising the possibility that NF- $K \mathrm{~B}$ has a part in perpetuating the infection and that viral genes activate $\mathrm{NF}-\kappa \mathrm{B}$. Several mechanisms could account for the increase in binding activity;

the induction of cellular protein kinases, transcriptional activation of p105 and/or $\mathrm{p} 65$, inhibition of $\mathrm{I}_{\kappa} \mathrm{B}$ synthesis or activation of a protease might all serve this function. Rivière et al. ${ }^{1}$ provide evidence for an intriguing possibility: the HIV protease, thought normally to regulate maturation of the virus by cleavage of the HIV polyprotein within budding forms, might also contribute to NF- $\kappa \mathrm{B}$ activation. They have detected a smaller form of the processed p105 DNAbinding subunit. This smaller protein, of $M$ $45 \mathrm{~K}$, is also generated by cotransfection with the HIV protease, and a potential aspartyl protease cleavage site has been identified which could give rise to this protein. Incubation of $\mathrm{p} 105 \mathrm{NF}-\kappa \mathrm{B}$ with the HIV protease in vitro also generates the protein. Normally, a yet undefined cellular protease is likely to serve this function.

These findings are an elegant example of the precision of viral/host cell interactions, and raise additional questions about the molecular activation of HIV during infection and the role of $\mathrm{NF}-\kappa \mathrm{B}$. The HIV protease has been thought to be confined to the budding virus particle. This assumption must now be reassessed. Another possibility is that the virus particle can deliver sufficient protease to the cell to stimulate proteolysis of p105. At the same time, the possibility remains that HIV activates other cellular proteins, including protein kinases, cellular

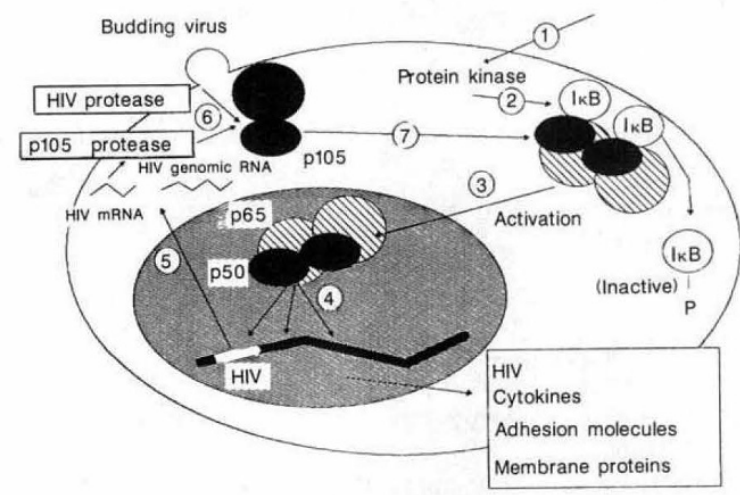

Mechanism of NF- $\kappa \mathrm{B}$ activation and its relationship to HIV infection. (1) Cells are activated by cytokines or mitogens. (2) Cellular protein kinases are induced, causing phosphorylation of $I_{K} B$ and other potential regulatory proteins. The phosphorylated form of $\mid \kappa B$ does not complex to $p 50 / p 65$. (3) Dissociation of $\mathrm{p} 50 / \mathrm{p} 65$ from $\left.\right|_{\kappa} \mathrm{B}$ allows its translocation to the nucleus. (4) Nuclear NF- $k$ B stimulates increased transcription of HIV and other cellular genes, including those responsible for cytokines, adhesion molecules and cell-surface glycoproteins. RNA (5) Viral RNA is synthesized, including messenger RNAs encoding structural proteins, such as the HIV protease, and genomic HIV RNA. (6) Activation of the HIV protease stimulates cleavage of the p105 precursor of the DNA-binding subunit of NF- $\kappa$ B. Infection or cellular activation could also induce cellular proteases, including the endogenous $p 105$ protease. (7) The p50 DNAbinding subunit is incorporated into the $p 50 / p 65$ complex. 10 and 11; M.-L. Hammarskjold, personal communication).

Apparently, there is a common theme in infection by these different viruses which is not yet understood. As viral genes help to define critical steps in the regulation of cellular gene expression, these new insights may allow us to learn what the virus has already discovered.

Gary J. Nabel is in the Howard Hughes Medical Institute, University of Michigan Medical Center, Ann Arbor, Michigan 48109, USA.

\footnotetext{
1. Rivière, Y., Blank, V., Kourilsky, P. \& Israel, A. Nature 350 625-626 (1991)

2. Bachelerie, F., Alcami, J., Arenzana-Seisdedos, F. \& Virelizier, J.L. Nature 350, 709-712 (1991).

3. Nabel, G. \& Baltimore, D. Nature 326, 711-713 (1987)

4. Zagury, D. et al. Science 231, 850-853 (1986).

5. Harada, S. et al. Virology 154, 249-258 (1986)

6. Leonard, J. et al. J. Virol. 63, 4919-4924 (1989)

7. Ghosh, S. \& Baltimore, D. Nature 344, 678-682(1990)

8. Poli, G. et al. Proc. natn. Acad. Sci. U.S.A. 87, 782-785

(1990).

9. Gimble, J. M. et al. J. Virol. 62, 4104-4112 (1988).

10. Leung, K. \& Nabel, G. J. Nature 333, 776-778 (1988)

11. Ballard, D. W. et al. Science 241, 1652-1655 (1988).
} 